\title{
An X-ray diffraction study of defect parameters in a Ti-base alloy
}

\author{
G KARMAKER, P MUKHERJEE ${ }^{\dagger}$, A K MEIKAP, S K CHATTOPADHYAY and \\ S K CHATTERJEE*
}

Regional Engineering College, Durgapur 713 209, India

${ }^{\dagger}$ Variable Energy Cyclotron Centre, 1/AF, Bidhan Nagar, Calcutta 700 064, India

MS received 6 August 2001

\begin{abstract}
Detailed studies based on the well established method of Fourier line shape analysis have been made on the X-ray diffraction profile of hexagonal titanium alloy of nominal composition Ti-6.58\% Al-3.16\% Mo-1.81\% Zr-0.08\% Fe-0.012\% N-0.0078\% C. While deformation fault probability, $\alpha$, has been found to be quite high compared to that of pure titanium, the deformation growth fault parameter, $\beta$, shows a negative value ruling out the presence of growth fault in this alloy in the deformed state.
\end{abstract}

Keywords. Cold working; XRD; titanium alloy; microstructural parameters.

\section{Introduction}

The X-ray diffraction study of microstructural parameters to reveal the nature of imperfections in metallic alloys is an established technique and is also finding wide applications in the recent studies of cold-worked alloys and nanocrystalline materials (Adler and Wagner 1962; Delhez et al 1982; De and Sengupta 1984; Van Berkum et al 1993, 1994). Titanium alloys are used extensively in aviation industry as suitable structural material offering an excellent combination of strength and ductility. The present material has been chosen to investigate as to how the fault parameters control the deformation characteristics of this alloy. The microstructural parameters like coherent domain size, deformation fault parameter $\alpha$, growth fault parameter $\beta$ and micro strain $\left\langle\varepsilon_{\mathrm{L}}^{2}\right\rangle^{1 / 2}$ have been determined by considering a number of fault unaffected $(H-K=3 N)$ and fault affected $(H-K=3 N \pm 1)$ reflections.

\section{Experimental procedure and method of analysis}

A very drastic cold working was performed on the rod of the said composition procured from the Hindustan Aeronautics Ltd., Bangalore, with jewellery file at room temperature. The powdered samples were magnetically separated and sieved through a 250 mesh screen and flat diffractometer sample in the form of briquette suitable for the recording of XRD profile was prepared. A portion of the cold worked sample was vacuum sealed and annealed at $500^{\circ} \mathrm{C}$ for $10 \mathrm{~h}$ to annul the effect of cold work and

*Author for correspondence cooled in air for the preparation of the standard sample required to make the profile analysis.

The XRD profiles for both the cold worked specimen as well as the standard specimen were recorded with $\mathrm{CuK}_{\alpha}$ radiation in a highly stabilized Siemens Kristalloflex-4 X-ray Diffractometer operating at $30 \mathrm{kV}, 12 \mathrm{~mA}$ and having $1^{\circ}$ and $0.05 \mathrm{~mm}$ as divergence and receiving slits, respectively.

The detailed line profile analyses for hexagonal system (Warren 1969; Chatterjee and Sengupta 1975; Chattopadhyay et al 1990) have been performed making use of the following equations worked out by Warren and Averbach in their method of fourier analysis of imperfections in metallic alloys. The Stoke's corrected (De and Sengupta 1984; Warren 1969) fourier coefficients $A_{\mathrm{L}}$ are related to the size coefficients $A_{\mathrm{L}}^{\mathrm{S}}$ and distortion coefficients $A_{\mathrm{L}}^{\mathrm{D}}$ by

$$
A_{\mathrm{L}}=A_{\mathrm{L}}^{\mathrm{S}} A_{\mathrm{L}}^{\mathrm{D}},
$$

which can be separated in $A_{\mathrm{L}}^{\mathrm{S}}$ and $A_{\mathrm{L}}^{\mathrm{D}}$ from the $\log$ plot of $A_{\mathrm{L}}$ vs $1 / d^{2}$ where $d$ is the interplanar spacing (Warren 1969). Assuming Gaussian distribution of strain, the distortion coefficient $A_{\mathrm{L}}^{\mathrm{D}}$ is given by

$$
A_{\mathrm{L}}^{\mathrm{D}}=\exp \left(-2 \pi^{2} L^{2}<\varepsilon_{\mathrm{L}}^{2}>/ d^{2}\right),
$$

where $L=n a_{3}^{\prime}$ i.e. undistorted distance between the cells and $a_{3}^{\prime}=\lambda /\left[2\left(\sin \theta_{2}-\sin \theta_{1}\right)\right]$, where $\theta_{2}$ and $\theta_{1}$ are the truncation limits equispaced around $2 \theta_{0}$ (peak position) within which the profiles are expressed as fourier series (Warren 1969) and $n$ the harmonic number. For fault unaffected reflections $(H-K=3 N)$, where $H-K L_{0}$ are the hexagonal indices and initial slope of $A_{\mathrm{L}}^{\mathrm{S}}$ vs $L$ is related as

$$
-\left[\mathrm{d} A_{\mathrm{L}}^{\mathrm{S}} / \mathrm{d} L\right]_{\mathrm{L} \rightarrow 0}=1 / D,
$$

where $D$ is the average domain size (Warren 1969). The 
Table 1. Values of lattice parameters $a$ and $c$, average domain size, $D$ and r.m.s. strain $\left\langle\varepsilon_{\mathrm{L}}^{2}\right\rangle^{1 / 2}$ for Ti-base alloy.

\begin{tabular}{|c|c|c|c|c|c|}
\hline \multirow{2}{*}{$\begin{array}{l}\text { Alloy } \\
\text { composition }\end{array}$} & \multicolumn{2}{|c|}{ Lattice parameters } & \multirow{2}{*}{$\begin{array}{l}\text { Fault unaffected } \\
\text { reflections } \\
H-K=3 N\end{array}$} & \multirow[b]{2}{*}{$D(\AA)$} & \multirow{2}{*}{$\begin{array}{c}\text { r.m.s. strain } \\
<\varepsilon_{\mathrm{L}}^{1}>^{1 / 2} \text { at } \\
L=50 \AA\end{array}$} \\
\hline & $a(\AA)$ & $c(\AA)$ & & & \\
\hline $\mathrm{Ti}-88 \cdot 3502 \%$ & $3 \cdot 2367$ & $5 \cdot 1581$ & $11 \cdot 0$ & 675 & $5 \cdot 3 \times 10^{-3}$ \\
\hline $\mathrm{Al}-6 \cdot 58$ & & & $00 \cdot 2$ & & \\
\hline Мo-3·16 & & & $01 \cdot 0$ & & \\
\hline $\mathrm{Zr}-1 \cdot 81$ & & & & & \\
\hline $\mathrm{Fe}-0.08$ & & & & & \\
\hline $\mathrm{Ni}-0 \cdot 012$ & & & & & \\
\hline C-0.0078 & & & & & \\
\hline
\end{tabular}

slope of $\log A_{\mathrm{L}}$ vs $1 / d^{2}$ gives the measurement of microstrain $\left\langle\varepsilon_{\mathrm{L}}^{2}\right\rangle$ for various values of $L$.

Assuming the average domain size, $D$ and micro strain $\left\langle\varepsilon_{\mathrm{L}}^{2}\right\rangle$ thus determined from fault free reflections to remain uniform, the distortion coefficients $\left(A_{\mathrm{L}}^{\mathrm{D}}\right)$ for faulted reflections are calculated using (2) and size coefficients $\left(A_{\mathrm{L}}^{\mathrm{S}}\right)$ for faulted reflections are separated from Stoke's corrected fourier coefficients $A_{\mathrm{L}}$ for faulted $(H-K=$ $3 N \pm 1, L_{0}$ odd or even) reflections (1). The initial slope of these $A_{\mathrm{L}}^{\mathrm{S}}$ vs $L$ is then given as (Warren 1969; Chatterjee and Sengupta 1975; Van Berkum et al 1994)

$$
\begin{aligned}
-\left[\mathrm{d} A_{\mathrm{L}}^{\mathrm{S}} / \mathrm{d} L\right]_{\mathrm{L} \rightarrow 0}= & 1 / D_{\mathrm{e}}=1 / D+\left|L_{0}\right| d(3 \alpha+\beta) / C^{2} \\
& \text { for } L_{0} \text { even, } \\
-\left[\mathrm{d} A_{\mathrm{L}}^{\mathrm{S}} / \mathrm{d} L\right]_{\mathrm{L} \rightarrow 0}= & 1 / D_{\mathrm{e}}=1 / D+\left|L_{0}\right| d(3 \alpha+3 \beta) / C^{2} \\
& \text { for } L_{0} \text { odd, }
\end{aligned}
$$

where $D_{\mathrm{e}}$ is the effective domain size in the faulted planes, $D$ the average domain size obtained from fault free reflections, $\alpha$ and $\beta$ are respectively the deformation fault and growth fault parameters, and $c$ the hexagonal axis $\left(c=2 d_{00 \cdot 2}\right)$.

\section{Results and discussion}

The nominal composition of the titanium base alloy under investigation is Ti-6.58\% $\mathrm{Al}-3.16 \% \mathrm{Mo}-1.81 \% \mathrm{Zr}-$ $0.08 \% \mathrm{Fe}-0.012 \% \mathrm{Ni}-0.0078 \%$ C. From the peak position $\left(2 \theta_{0}\right)$ for all the observable reflections both belonging to fault unaffected and fault affected reflections, the lattice parameters have been calculated and are shown in table 1 . This shows a cla ratio of 1.594 which is same as other Ti-base alloys studied before (Sen et al 1995, 1997, 1998) and also as pure titanium (Chatterjee and Sengupta 1975).

The Stoke's corrected fourier coefficients $A_{\mathrm{L}}$ for fault unaffected $(H-K=3 N)$ and fault affected $(H-K=3 N \pm 1)$ for $L_{0}$ odd or even are shown in figures 1 and 2 , respectively. The $A_{\mathrm{L}}^{\mathrm{S}}$ and $A_{\mathrm{L}}^{\mathrm{D}}$ coefficients $\left(A_{\mathrm{L}}=A_{\mathrm{L}}^{\mathrm{S}} A_{\mathrm{L}}^{\mathrm{D}}\right)$ have been separated from the plot of $\log A_{\mathrm{L}}$ vs $1 / d^{2}$ for fault unaffected reflections. The plot of $\log A_{\mathrm{L}}$ vs $1 / d^{2}$ is shown in figure 3 . The intercepts give $A_{\mathrm{L}}^{\mathrm{S}}$ and slope gives the

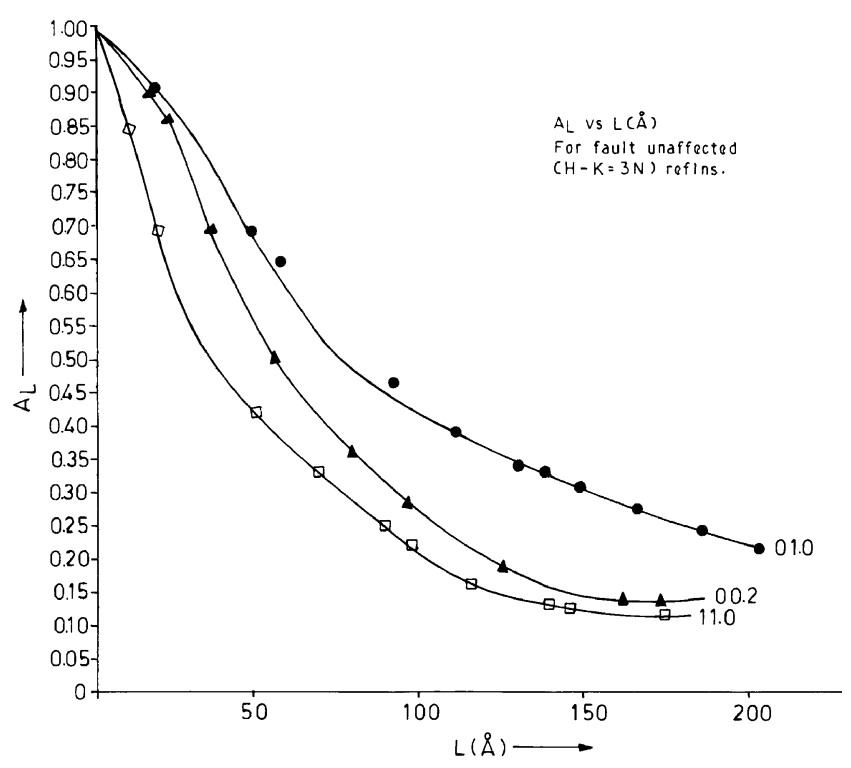

Figure 1. $A_{\mathrm{L}}$ vs $L(\AA)$ for fault unaffected $H-K=3 N$ reflections.

strain. The average domain size, $D$ calculated by using (3) from $A_{\mathrm{L}}^{\mathrm{S}}$ of fault free $H-K=3 N$ reflections is obtained as $675 \AA$, which is slightly higher than that of pure titanium (Chatterjee and Sengupta 1975) and is of the same order as those for titanium alloys (Sen et al 1995, 1997, 1998). The r.m.s. strain $\left\langle\varepsilon_{\mathrm{L}}^{2}\right\rangle^{1 / 2}$ has been calculated from the slope of $\log A_{\mathrm{L}}$ vs $1 / d^{2}$ for fault unaffected reflections and its variation with $L$ is shown in figure 4 . The value of $\left\langle\varepsilon_{\mathrm{L}}^{2}\right\rangle^{1 / 2}$ at $L=50 \AA$ is $5.35 \times 10^{-3}$, which is also slightly higher than that for pure titanium but of the same order as that for titanium alloys (Sen et al 1995, 1997, 1998). Assuming the r.m.s. strain $\left\langle\varepsilon_{\mathrm{L}}^{2}\right\rangle^{1 / 2}$ to be uniform both for fault unaffected and fault affected reflections, the $A_{\mathrm{L}}^{\mathrm{D}}$ values of fault affected reflections were calculated and the $A_{\mathrm{L}}^{\mathrm{S}}$ coefficients have been sorted out from $A_{\mathrm{L}}$ coefficients of fault affected reflections. The plot of $A_{\mathrm{L}}^{\mathrm{S}}$ vs $L$ for fault affected reflections is shown in figure 5 . The initial slopes of $A_{\mathrm{L}}^{\mathrm{S}}$ vs $L$ give corresponding effective domain size, $D_{\mathrm{e}}$ $(\AA)$. The values of $D_{\mathrm{e}}$ are listed in table 2 along with the compound fault parameters $(3 \alpha+\beta)$ when $L_{0}$ is odd and 
$(3 \alpha+3 \beta)$ when $L_{0}$ is even. The effective domain sizes are found to be sufficiently low indicating the presence of deformation faults. The deformation fault parameter $\alpha$ and growth fault parameter $\beta$ have been calculated from the compound fault parameter values for various reflec-

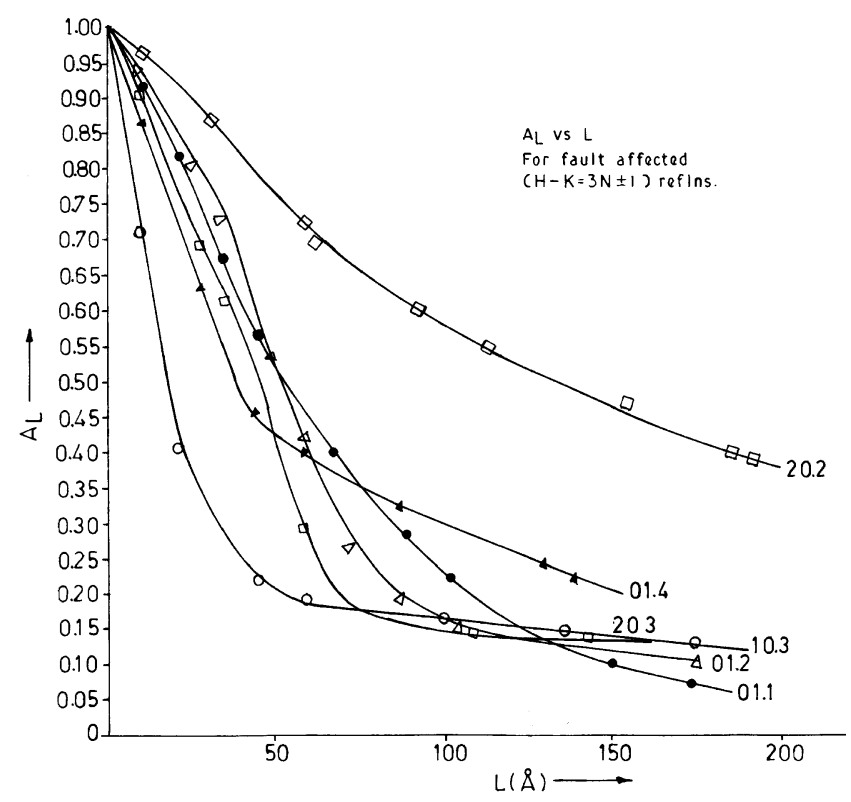

Figure 2. $A_{\mathrm{L}}$ vs $L(\AA)$ for fault affected $H-K=3 N \pm 1\left(L_{0}\right.$ even and odd) reflections.

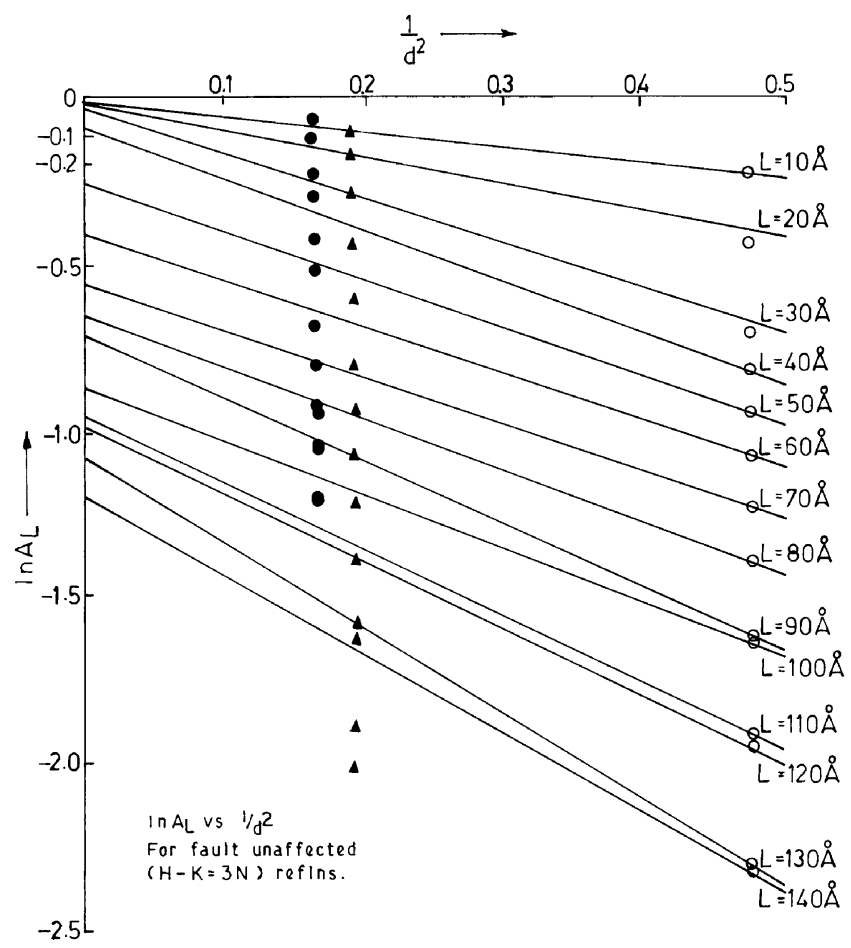

Figure 3. $\ln A_{\mathrm{L}}$ vs $1 / d^{2}$ for fault unaffected reflections. tions and are shown in table 2. It has been found that the value of $\alpha$ is quite high for this alloy compared to that of pure titanium (Chatterjee and Sengupta 1975). The increase in the value of $\alpha$ from pure titanium by an order of magnitude (from $8.1 \times 10^{-3}$ to $103 \times 10^{-3}$ ) is due to the

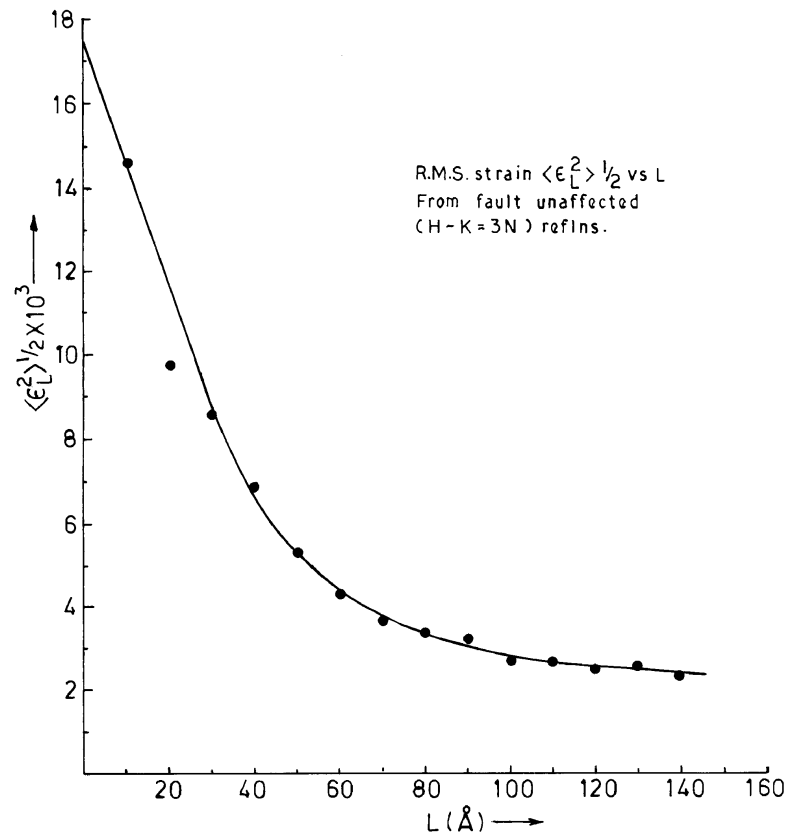

Figure 4. $\left\langle\varepsilon_{\mathrm{L}}^{2}\right\rangle^{1 / 2}$ vs $L(\AA)$ for fault unaffected reflections.

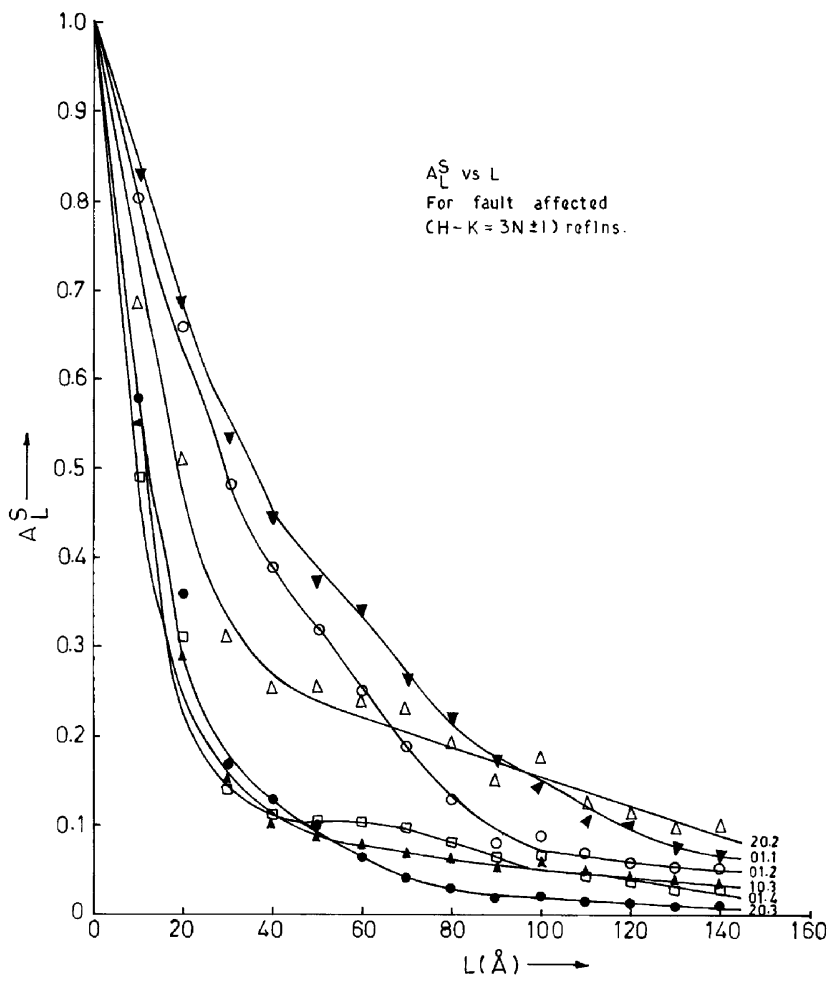

Figure 5. $A_{\mathrm{L}}^{\mathrm{S}}$ vs $L(\AA)$ for fault affected reflections. 
Table 2. Values of effective domain size, compound fault parameters, deformation fault, $\alpha$ and twin fault, $\beta$ parameters for Ti-base alloy.

\begin{tabular}{lccccc}
\hline & \multicolumn{4}{c}{$\begin{array}{c}\text { Compound fault } \\
\text { parameters }\left(\times 10^{3}\right)\end{array}$} & \\
\cline { 2 - 4 } $\begin{array}{l}\text { Fault affected } \\
\text { reflections }\end{array}$ & & $3 \alpha+\beta$ & $3 \alpha+3 \beta$ & & \\
$H-K=3 N \pm 1$ & $D_{\mathrm{e}}(\AA)$ & $L_{0}=$ odd & $L_{0}=$ even & $\alpha \times 10^{3}$ & $\beta \times 10^{3}$ \\
\hline $20 \cdot 3$ & $24 \cdot 0$ & $362 \cdot 8$ & & $103 \cdot 4$ & $-37 \cdot 1$ \\
$10 \cdot 3$ & $23 \cdot 0$ & $281 \cdot 2$ & & & \\
$01 \cdot 1$ & $62 \cdot 0$ & $175 \cdot 0$ & & & \\
$01 \cdot 2$ & $49 \cdot 0$ & & $146 \cdot 71$ & & \\
$20 \cdot 2$ & $37 \cdot 0$ & & 299.97 & & \\
01.4 & 19.5 & & 312.50 & & \\
\hline
\end{tabular}

effect of solute. But when the value of $\alpha$ obtained for this alloy is compared with that in $\mathrm{Ti}-\mathrm{Al}\left(\sim 90 \times 10^{-3}\right)$ and $\mathrm{Ti}-\mathrm{Zr}\left(\sim 100 \times 10^{-3}\right)$, it is found that the usual effect of $\mathrm{Al}$ as solute which is to increase the faulting tendency undergoes a reversal in the presence of other solute elements like b.c.c. Mo (3.16 wt.\%) and shows a decreasing trend. It is known that h.c.p. structure while undergoing faulting, the close packed basal planes change the stacking sequence from $\mathrm{AB} \mathrm{AB} \ldots$ to $\mathrm{ABC} \mathrm{ABC} \ldots$ similar to that for the stacking sequence of (111) planes in f.c.c. systems. The addition of $\mathrm{Al}$ (f.c.c.) has been observed to enhance the formation of this faulting sequence as in $\mathrm{Ti}-\mathrm{Al}$ alloy (Sen et al 1995, 1998), and zirconium also lowers the stacking fault energy in titanium due to their size difference (Sen et al 1998). In the present alloy where both $\mathrm{Al}$ and $\mathrm{Zr}$ are present as alloying elements, their additive effect towards the increase of deformation faulting is not as pronounced as expected. This may be due to the presence of Mo (b.c.c.), whose sole effect is probably to suppress the faulting and increase the stacking fault energy to some extent. The growth fault parameter $\beta$ has been observed to be negative which is similar to the earlier cases for pure titanium and also $\mathrm{Ti}$ base $\mathrm{Al}$ and $\mathrm{Zr}$ alloys. This shows that growth faults are totally absent in this hexagonal system as is also observed in other hexagonal structures (Chatterjee and Sengupta 1975; Chattopadhyay et al 1990; Sen et al 1995, 1997, 1998). So, this alloy also, like other titanium base alloys investigated by similar technique (Sen et al 1995, 1998) can be effectively used in the aviation industry due to its rapid strain hardenability.

\section{Conclusion}

Alloying of titanium with $\mathrm{Al}$ and $\mathrm{Zr}$ increased its deformation faulting tendency. The growth fault parameter $\beta$ however, was negligible as was observed in various other hexagonal systems.

\section{References}

Adler R P I and Wagner C N J 1962 J. Appl. Phys. 333451

Chatterjee S K and Sen Gupta S P 1975 J. Mater. Sci. 101093

Chattopadhyay S K, Chatterjee S K and Sen Gupta S P 1990 J. Mater. Res. 52120

Delhez R, de Keijser Th H and Mittemeijer E J 1982 Fresnius Z Anzl. Chem. 3121

De M and Sen Gupta S P 1984 Pramana - J. Phys. 23721

Sen R, Chattopadhyay S K and Chatterjee S K 1995 Trans. Indian Inst. Metals $\mathbf{4 8} 247$

Sen R, Chattopadhyay S K and Chatterjee S K 1997 Jpn. J. Appl. Phys. 36364

Sen R, Chattopadhyay S K and Chatterjee S K 1998 Met. \& Mater. Trans. A29 2639

Van Berkum J G M, Vermeulen A C, Delhez R, de Keijser Th H and Mittemeijer E J 1993 Mater. Sci. Forum 13377

Van Berkum J G M, Vermeulen A C, Delhez R, de Keijser Th H and Mittemeijer E J 1994 J. Appl. Cryst. 27345

Warren B E (ed.) 1969 X-ray diffraction (MA, Reading: Addison Wesley) 